\title{
The compound stream event of March 20-25, 2011 as measured by the STEREO B spacecraft
}

\author{
Duraid A. Al-Shakarchi ${ }^{1,2} \cdot$ Huw Morgan ${ }^{2}$
}

Received: 7 December 2019 / Accepted: 26 March 2020 / Published online: 1 April 2020

(c) The Author(s) 2020

\begin{abstract}
The interaction of interplanetary coronal mass ejections (ICME) with each other and with co-rotating interaction regions (CIR) changes their configuration, dynamics, magnetic field and plasma characteristics and can make space weather forecasting difficult. During the period of March 20-25, 2011, the Solar Terrestrial Relation Observatory (STEREO B) encountered a compound stream containing several interacting structures. Our analysis suggests that the stream consists of two ICMEs followed by an embedded ICME/CIR. The sudden appearance of the third ICME within the fast wind side of the CIR causes the proton temperature $\left(T_{p}\right)$ to drop suddenly to its lowest level in about 1.2 hours, from $3.89 \times 10^{5} \mathrm{~K}$ to $1.07 \times 10^{4} \mathrm{~K}$ (by a factor of $\approx 36$ ). The fast wind which follows the CIR influences not only the third ICME's temperature but also its proton beta $\left(\beta_{\mathrm{p}}\right)$. In addition, the third ICME impacts the CIR through expansion and deceleration. A forward pressure wave penetrates ICME1. It's source is either the second ICME, the merged third ICME and CIR, or it may be a remnant shock. The compression causes an increase of the $T_{p}$ and $\beta_{\mathrm{p}}$ of the second and part of the first ICME. Despite the signatures of four large-scale interacting structures within the compound stream, it is difficult to reconcile the in-situ sequence with other remote sensing observations of ejecta close to the Sun because of the large system of coronal expanding loops above the active region. Compound streams therefore remain difficult to interpret, and further understanding of the subject will depend on the future study of similar events.
\end{abstract}

H. Morgan

1 Department of Astronomy \& Space, University of Baghdad, Baghdad, Iraq

2 Department of Physics, Aberystwyth University, Ceredigion, SY23 3BZ, Wales, UK
Keywords Interplanetary coronal mass ejection (ICME) . Co-rotating interaction region $\cdot$ Stream interface (SI), and compound stream

\section{Introduction}

During their propagation through interplanetary space, ICMEs can interact either with each other or with CIRs, making space weather forecasting more difficult. This interaction can alter their configuration, dynamics, and their magnetic field and plasma characteristics (Winslow et al. 2016; Al-Shakarchi 2018), including flux rope deformations, reverse shock formations (Lugaz et al. 2005; AlShakarchi and Morgan 2018), radio emission enhancements, increases in $T_{p}$, and ICME deflections (Lugaz et al. 2012; Liu et al. 2012; Oliveros et al. 2012; Farrugia et al. 2012; Lugaz et al. 2017; Shen et al. 2012).

At $1 \mathrm{AU}$, interplanetary streams are classified as either "simple", "irregular", or "compound" (Burlaga and Ogilvie 1972; Burlaga 1975). Burlaga et al. (1986) defined a compound stream as the interaction between a fast and slow stream. Between 0.85 and $6.2 \mathrm{AU}$, they found that a compound stream formed as a consequence of the interaction between, and the coalescence of, two exceptionally fast transient streams overtaking two co-rotating streams and a slow transient stream. They defined a fast stream as an interplanetary flow with speed greater than $475 \mathrm{~km} / \mathrm{s}$. Furthermore, Burlaga et al. (1987) suggested that compound streams are formed by the interaction of two or more fast interplanetary ejecta, with fast being defined as greater than $450 \mathrm{~km} / \mathrm{s}$. They also state there should be at least two interaction regions (two magnetic field magnitude peaks) present. Their study includes many examples of interactions between different transient ejecta, or between transient ejecta and co- 
rotating streams, and described the results of these interactions in terms of compound streams. During the period of 1972-1983, nine out of 17 intense magnetic storms $\left(A_{p}>\right.$ 90) occurred as a consequence of amplified magnetic magnitudes as a result of structures interacting within compound streams. Both magnetic field strengths and densities were higher than for isolated flows. Jian et al. (2008) identified many hybrid CIR-ICME events and found that their structures sometimes possessed an irregular profile or some complexity in addition to their original morphology. Cane and Richardson (2003) found that only about $8 \%$ of ICMEs were identified within the High-Speed Stream (HSS) passage at 1 AU. Possibly due to the ICME sources always being located far away from the weak field regions, the ICMEs are infrequently embedded within HSS (Forsyth et al. 2006). Moreover, ICMEs were found more frequently at the leading front of the HSS or within the slow interstream solar wind. The CIR which follows the ICME is able to overtake the trailing region of the ICME, compressing the rear region and deforming the magnetic cloud (MC) flux rope.

Burlaga et al. (2001) classified the faster ejecta into magnetic clouds and complex ejecta. They defined complex ejecta as fast non-co-rotating interplanetary flows ( $>600 \mathrm{~km} / \mathrm{s}$ ) with a disturbed magnetic field (not flux ropes) and an average $\beta_{p}$ of $0.25 \pm 0.09$, which is greater than that found in MCs. The features of the individual CMEs disappear as the interaction progresses towards 1 AU (Burlaga et al. 2002). On the other hand, Wang et al. (2003) considered multiple MCs overtaking each other due to successive CMEs as complex structures. They concluded that each subcloud has characteristics that are consistent with isolated MCs except for $T_{p}$, due to the sub cloud compression and a rising $\beta_{\mathrm{p}}$ in the interaction regions.

The formation of ICME-ICME and ICME-CIR interactions within compound streams takes many forms. Sometimes ICME-driven shocks interact with each other without ejecta interacting, or the two ejecta interact with each other and an associated magnetic reconnection exhaust region appears between them as a result of these different interacting structures. Sometimes the ICME-driven shock which follows blows through the preceding ICME and interacts with its ejecta (Lugaz et al. 2015; Mishra et al. 2015; Liu et al. 2012). The consequence of these interactions lead to an enhancement of the southward geomagnetic field which occur in intense magnetic storms (Farrugia and Berdichevsky 2004; Farrugia et al. 2006; Liu et al. 2015).

Throughout the solar cycle duration, the rate of large CMEs increases from 2-3 per week at solar minimum to 5-6 per day at solar maximum. Therefore, ICME-ICME interactions occur more frequently (but not exclusively) at solar maximum, making space weather forecasting difficult. Interacting ICMEs may originate from different source regions near the Sun, or from the same region: remote and in situ observations reveal that many ICME-ICME interactions occur due to recurrent CMEs which are launched from the same solar active region.

Tracking the propagation of CMEs from their launch at the Sun and through interplanetary space leads to a better understanding of the interaction of ICMEs with each other, the solar wind, and other structures, and also allows improved predictions of their arrival time at Earth (Mishra et al. 2015). The launch of the Solar Terrestrial Relations Observatory (STEREO, (Kaiser 2005)) enabled this tracking by providing continuous imaging from instruments including the $\mathrm{He}$ liospheric Imagers (HI) on board The Sun Earth Connection Coronal and Heliospheric Investigation (SECCHI, Howard et al. (2002)). Recently, many ICME interaction events have been studied by combining in situ measurements with wide angle point of view observations from the STEREO HIs; examples include the May 23-24, 2010 event (Lugaz et al. 2012), the August 1, 2010 event (Temmer et al. 2012; Harrison et al. 2012), and the February 13-15, 2011 event (Maričić et al. 2014; Temmer et al. 2014). Numerical modeling is an important factor in understanding ICME propagation and interaction (Prise et al. 2015; Winslow et al. 2016).

This paper is an observational study of an in-situ compound stream encountered by STEREO B (STB) over the period March 20 - 26, 2011. Sections 1 and 2 present remote sensing and in situ observations. Sections 3, 4 and 5 presents an analysis and interpretation of the results. The impact of the merged ICME/CIR is discussed in the last section.

\section{Remote sensing observations}

In this section, remote sensing observations from the 2011 March 17 active region are presented, which is the likely source of the 2011 March 20-25 in situ event. During the event period, the angular longitude separation (Heliocentric Earth equatorial, HEEQ) between STB and SOHO was between about $95.2^{\circ}$ to $95.3^{\circ}$. STB was located between about $6.7^{\circ}$ to $5.4^{\circ}$ south of SOHO. Figure $1 \mathrm{a}$ and b show the Extreme Ultraviolet Imager (EUVI/STB) $195 \AA$ images of the low corona. The images show two flares at 12:15 UT (left) and 14:15 UT (right) originating from the same active region.

Figure 2 shows a sequence of events as observed by the LASCO C2 (Brueckner et al. 1995) coronagraph. Before the launch of the large CME, there is a large system of expanding loops above an active region (indicated by the black ellipse). These loop systems are similar to those described by Morgan et al. (2013). This system of expanding loops is a precursor for a large CME originating from the same active region. This CME can first be seen in the top right panel of Fig. 2, as indicated by the green ellipse. Over the next few panels, the CME is seen to be a wide, complicated CME 


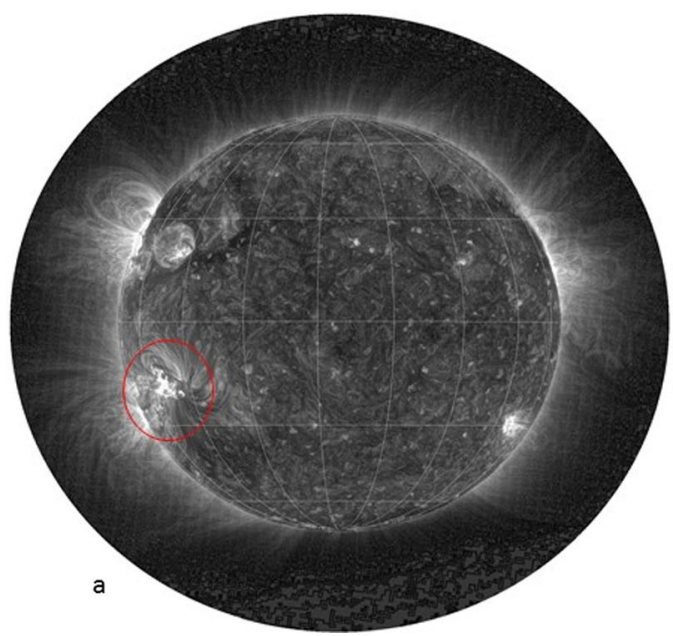

Fig. 1 STEREO B EUVI 195 A images, with an active region circled in red. The time of the images is 12:15 UT (left) and 14:15 UT (right), coinciding with the occurrence of two flares in the active region. These

containing several sub-structures. Some of the more prominent of these sub-structures are indicated by red and purple ellipses in the second and third row of Fig. 2. In the wake of the CME, several complicated ejecta can be seen, as indicated by the green, blue, yellow and other ellipses. The flare at 12:15 UT from the active region is clearly associated with the large CME (inside the dark blue ellipse). The large CME is mentioned in the STEREO A/B List of ICMEs (Jian et al. 2013) as a possible source of the in situ ICME which is described in the next section, but no reference is made to multiple ICMEs. Unfortunately, the STEREO B HI images do not show this large CME due to the viewing direction.

Figure 3 shows more detail of the active region that is the source of much of the activity seen in the coronagraph data, as observed by EUVI/STB in the $195 \AA$ channel. Following the 12:15 UT flare, the active region has two large separated loop systems (1 and 2). After the second flare at 14:15 UT, the outline of these loops becomes clearer, and then they gradually seem to merge into one arcade. The second flare is not associated with a large CME, but it is probably linked to smaller ejecta seen by LASCO C2 in the wake of the first large CME.

The presence of only one large CME in the remote sensing results seems to be inconsistent with the in-situ data that will be presented in the next section, which suggests the presence of two or more ICMEs. However, the remote sensing results do clearly show the complexity of the active source region, the system of expanding loops in front of the $\mathrm{CME}$, the complexity of the CME itself and the presence of ejecta following the CME.

Figure 4 shows a large CME observed on March 21, 2011, 15:36 UT by SOHO/ LASCO C2 with a linear speed of $963 \mathrm{~km} / \mathrm{s}$. The estimated transit time implies that it is the last in situ ICME of the compound event described in the

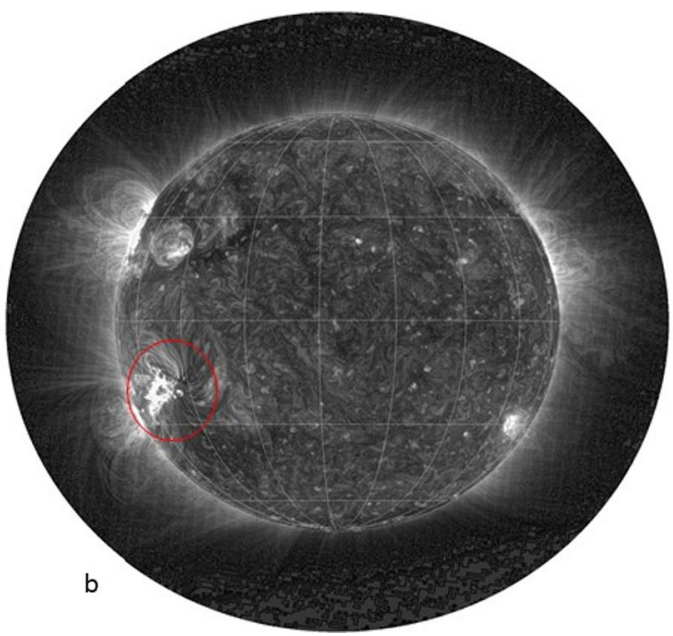

images have been processed using Multiscale Gaussian Normalization (Morgan and Druckmüller 2014)

next sections. A careful study of LASCO images for the 4 days leading up to the 17th did not reveal any other plausible candidate events that form this compound stream event.

\section{In situ observations}

Figure 5 displays the 1 min magnetic field and plasma measurements taken from the magnetometer (MAG) (Acuña et al. 2008) and the In Situ Measurements of Particles and CME Transient (IMPACT) instrument (Luhmann et al. 2008) onboard STEREO B over the period March 20, 00:00 UT to March 26, 00:00 UT. The suprathermal electron data was obtained by the Solar Wind Electron Analyser (SWEA) (Sauvaud et al. 2008). The event begins with a forward shock (S1) on March 20 at 17:20, manifesting an enhancement of the magnetic field magnitude, $T_{p}$, and a gradual elevation in proton density and speed. The duration between the 1st and 3rd vertical dotted lines is highly variable and complex, which implies the passage of a sheath region. Within the sheath, there is a large reduction in magnetic field strength (shaded yellow) for about three hours (March 21 00:30 - 04:10 UT) associated with high density and increasing $\beta_{\mathrm{p}}$. The heat flux distribution angle changed from $0^{\circ}$ to $180^{\circ}$, and a magnetic clock angle reversal (2nd vertical dotted line) indicates the presence of a heliospheric plasma sheet (HPS). The magnetic field strength then underwent a second reduction (March 21, 07:35 - 10:15 UT), with a large increase in density and $\beta_{\mathrm{p}}$ (higher than that of the surrounding), which is associated with a change in the magnetic field orientation.

The $18 \mathrm{hr}$ duration between the $3 \mathrm{rd}$ and 4 th vertical lines can be identified as a MC flux rope based on several criteria Zurbuchen and Richardson (2006): a drop in the $T_{p}$ and 

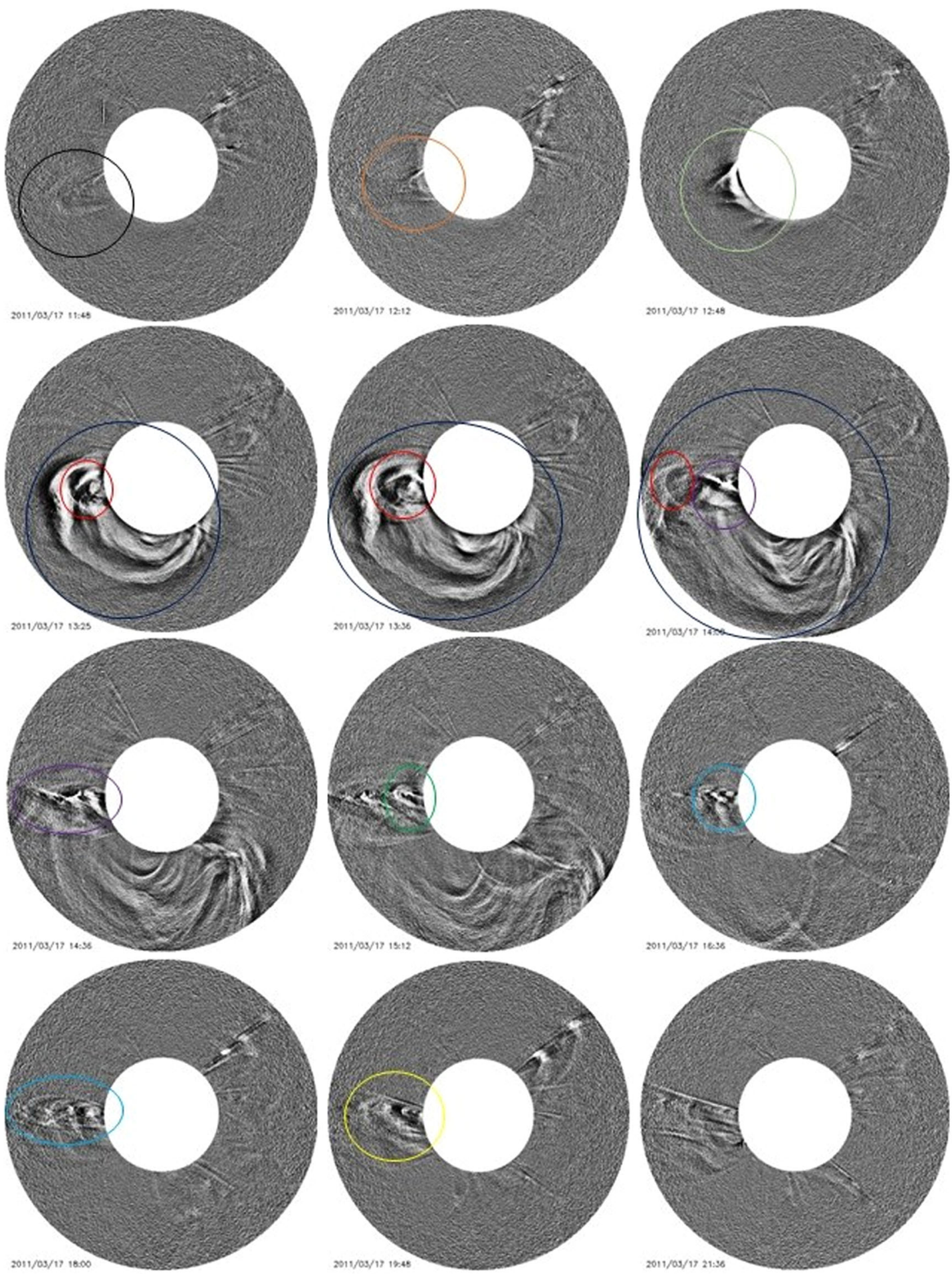

Fig. 2 SOHO/LASCO C2 images for 17-03-2011 (11:48 UT21:36 UT) displaying multiple dynamic features. Different coloured ellipses outline different events as described in the main text. These

$\beta_{\mathrm{p}}$ accompanied by a smooth coherent rotation of the magnetic field. Between the 4th and 5th vertical dotted lines, the coherent magnetic rotation becomes less smooth for about 12 hours, but it appears to be a continuation of the MC flux images have been processed to remove large-scale quiescent structures (i.e. streamers) using the Dynamic Separation Technique (Morgan et al. (2012), Morgan (2015))

rope (panel 5). The magnetic strength maintains its value in the range of 7.2-10.8 $\mathrm{nT}$ and the suprathermal electrons maintain their bidirectional distribution, implying a closed magnetic field region. The broad flat profile of the mag- 
Fig. 3 Further detail of the active region circled in red in Fig. 1 as observed by EUVI/STB in the $195 \AA$ channel. Two separated systems of coronal loops are labelled 1 and 2 and detailed in the main text
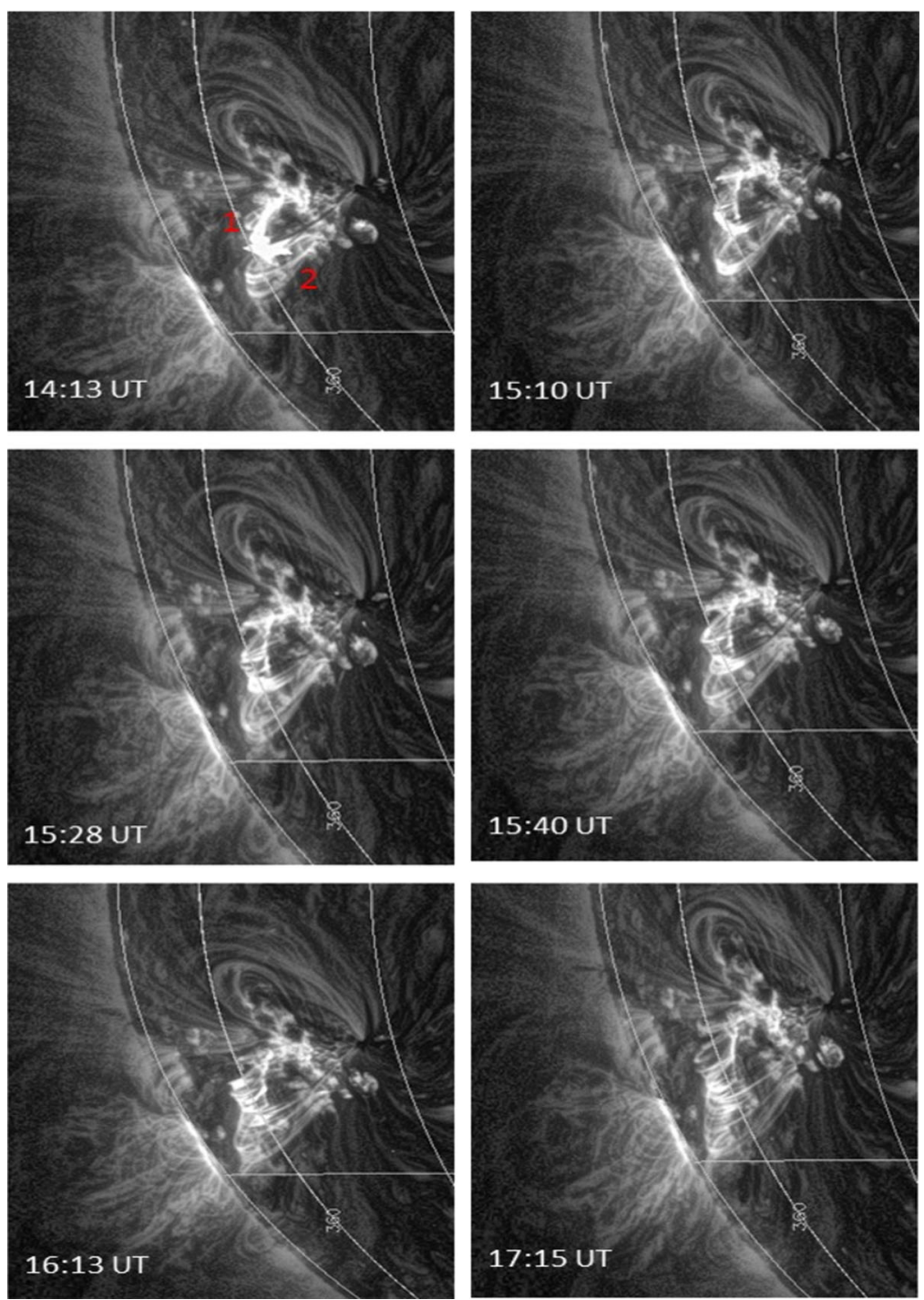

netic clock angle between the 3rd and 5th vertical lines suggests that the MC has somehow broken out of a larger ICME structure. This may be due to the long duration of the complex series of ejecta emerging from the active region in the wake of the initial large CME following the March $17 \mathrm{CME}$. This ICME will be referred to as ICME1.

Within the ICME1 flux rope, there is a noticeable shock structure (S2) which first appears on March 21, 18:40 UT, accompanied by a small but sharp increase in magnetic field magnitude (from 9.3 to $12.7 \mathrm{nT}$ ), an increase in proton speed $V_{p}$ from 445 to $487 \mathrm{~km} / \mathrm{s}$, a rise in $\mathrm{P}_{\mathrm{t}}$ (the sum of the magnetic pressure and thermal proton pressure (Russell et al. 2005)), and a short, transient elevation of the $T_{p}$. Unfortunately, there is a short data gap between 19:30 UT to 22:05 UT. Between March 22, 14:05-15:10 UT following ICME 1 the magnetic field magnitude decreases, associated with a heat flux discontinuity and a high increase in $\beta_{\mathrm{p}}, T_{p}, V_{p}$ and $\mathrm{P}_{\mathrm{t}}$ in comparison to the ambient solar wind. The duration between the 5th and 6th vertical lines is a magnetic reconnection exhaust region. During the interval between the 6 th and 7 th vertical lines, the magnetic field strength varies between 6.1 and $9.3 \mathrm{nT}$, associated with low $\beta_{\mathrm{p}}$ (average value 0.15 ) and very low density. The $194-$ $314 \mathrm{eV}$ suprathermal electron pitch angle distributions also display counter-streaming, associated with closed magnetic field lines. This leads us to consider this interval as another ICME, labelled ICME2. The dominant heat flux direction for both magnetic clouds (panel 1) rotates with the magnetic field (panel 5), causing an enhancement at $0^{\circ}$, consistent with the passage of the spacecraft close to the flank of both MCs (with both MC sides connected to the Sun).

After ICME2, there is a heat flux dropout, followed by a merged ICME-CIR (labelled ICME3). The CIR appears on March 23, 13:35 UT (7th vertical line), accompanied by an increase in solar wind speed from 394 to $466 \mathrm{~km} / \mathrm{s}$, 

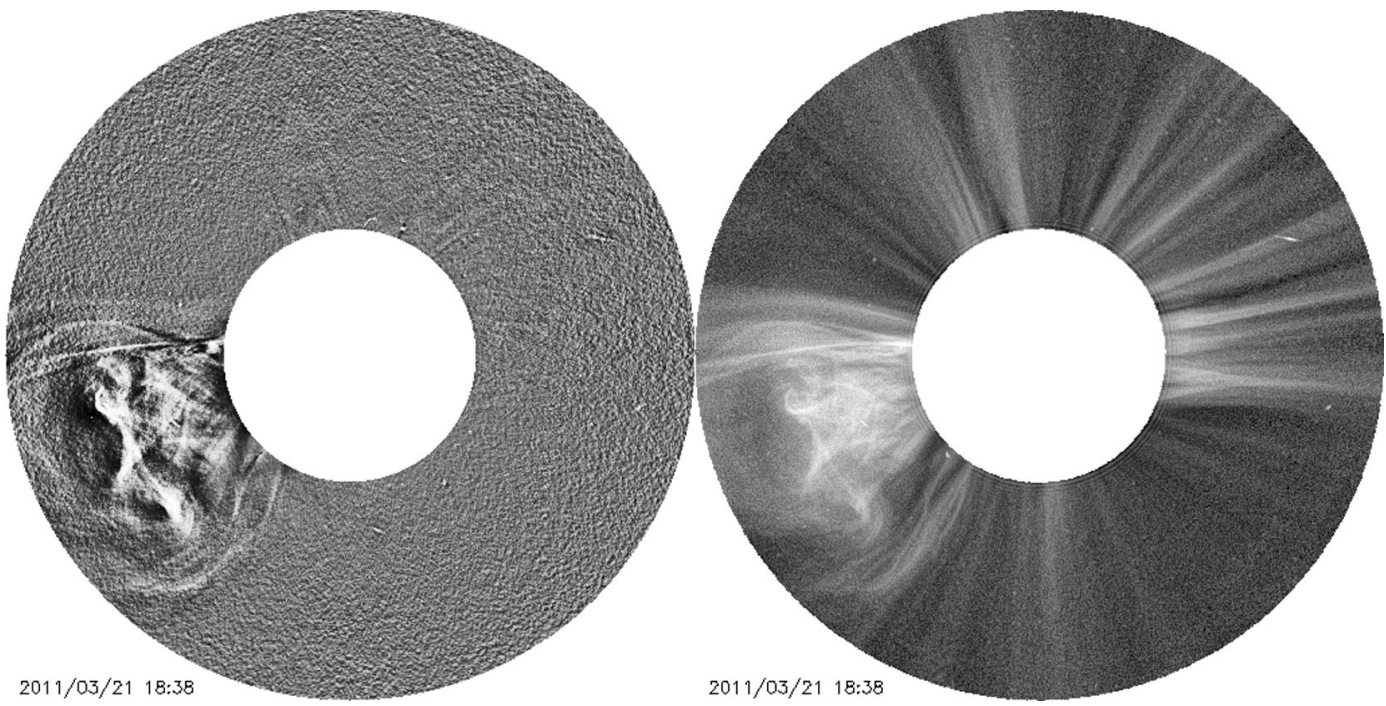

Fig. 4 SOHO/LASCO C2 image of the March 21, 2011, 15:36 UT large CME. The left image has been processed using a Dynamic Separation Technique (Morgan and Habbal 2010), and the right image has been processed using the Normalizing Radial Graded Filter of Morgan et al. (2006)

and a small rise in density and $\beta_{\mathrm{p}}$. A sudden drop in magnetic field magnitude (shaded green) represents the impact of the interaction region between ICME2 and CIR/ICME3. The magnetic field reduction reaches its lowest value at 3.7 $\mathrm{nT}$, which is associated with a sharp rise in proton density $N_{p}$ and $\beta_{\mathrm{p}}$, and a reversal of the magnetic clock angle (from 40 to 210 degrees), suggesting a magnetic reconnection exhaust region (Gosling et al. 2007; Xu et al. 2015). After this, the solar wind speed experiences a substantial yet gradual increase (March 23, 19:50 UT) with a significant jump in magnetic field strength, $P_{t}$, and enhanced heat flux at $0^{\circ}$, all suggesting the presence of a CIR. A stream interface (SI) feature appears at the 8 th vertical line $-\mathrm{P}_{\mathrm{t}}$ peaks with a gradual decline to both sides, and there is a drop-in density accompanied by an increase in temperature.

The signature of ICME3 begins around March 24, 05:10 UT (9th vertical line), identified by an initial non-smooth rotation in the $B_{n}$ component for about 4.5 hours, followed by a smooth and coherent magnetic field rotation until March 24, 17:30 UT—a duration of about 14 hours. This period has variable temperature, beginning with a considerable decrease then increase (for about three hours) and then a decrease that is less than the expected temperature $T_{e x}$ throughout the remainder of ICME3. $T_{e x}$ is the expected proton temperature obtained from Lopez (1987) and Richardson and Cane (1995). The period also exhibits low proton beta and bidirectional suprathermal electron pitch angle distribution. After March 24, 17:30 UT, following an initial variation, $B_{n}$ maintains its rotation until the 10th vertical dotted line (March 25, 00:50 UT).

\section{Pressure wave analysis}

The STB March 20 event is composed of several interplanetary structures (three ICMEs and a CIR). Their magnetic and plasma profiles can be distinguished, and they satisfy the definition of a compound stream (Burlaga et al. 1987), from which it can be deduced that they are not complex ejecta (Burlaga et al. 2002). The ICME boundaries are identified based on discontinuities in the magnetic field, heat flux, $T_{p}$, $\beta_{\mathrm{p}}$, and density (Fig. 5). The structural interactions may have a considerable effect on the $T_{p}$ profile. $\beta_{\mathrm{p}}$ will therefore be used here as the main parameter for identifying the ICME boundaries (Liu et al. 2012). The appearance of the heliospheric current sheet (HCS) (2nd vertical dotted line) within the sheath is a temporary change of the interplanetary magnetic field sector boundary during the passage of ICME1, the ICME therefore disrupts the coronal streamer belt locally(Zhao and Hoeksema 1996).

Figure 6 displays the magnetic and solar wind profiles for the duration of March 20, 12:00 UT-March 24, 00:00 UT. The figure illustrates two different interplanetary structures, ICME1 and ICME2, which are separated by a magnetic reconnection exhaust region, which suggests that ICME1 and ICME2 do not merge. Their similarity in size and energy may be the main reason why this event is so clearly a multiple ICME event (Lugaz et al. 2017). Within ICME1, S2 (Fig. 6, 3rd vertical dotted line) is similar to a pressure wave because the Alfven speed is about $185 \mathrm{~km} / \mathrm{s}$ and the proton speed increase is less than $50 \mathrm{~km} \mathrm{~s}^{-1}$ with Mach number $M_{A}=2.3$.

The solar wind profiles suggest many possible sources for the S2 feature. The first possibility is the trailing ICME (ICME2). Both the density and proton beta morphology sup- 


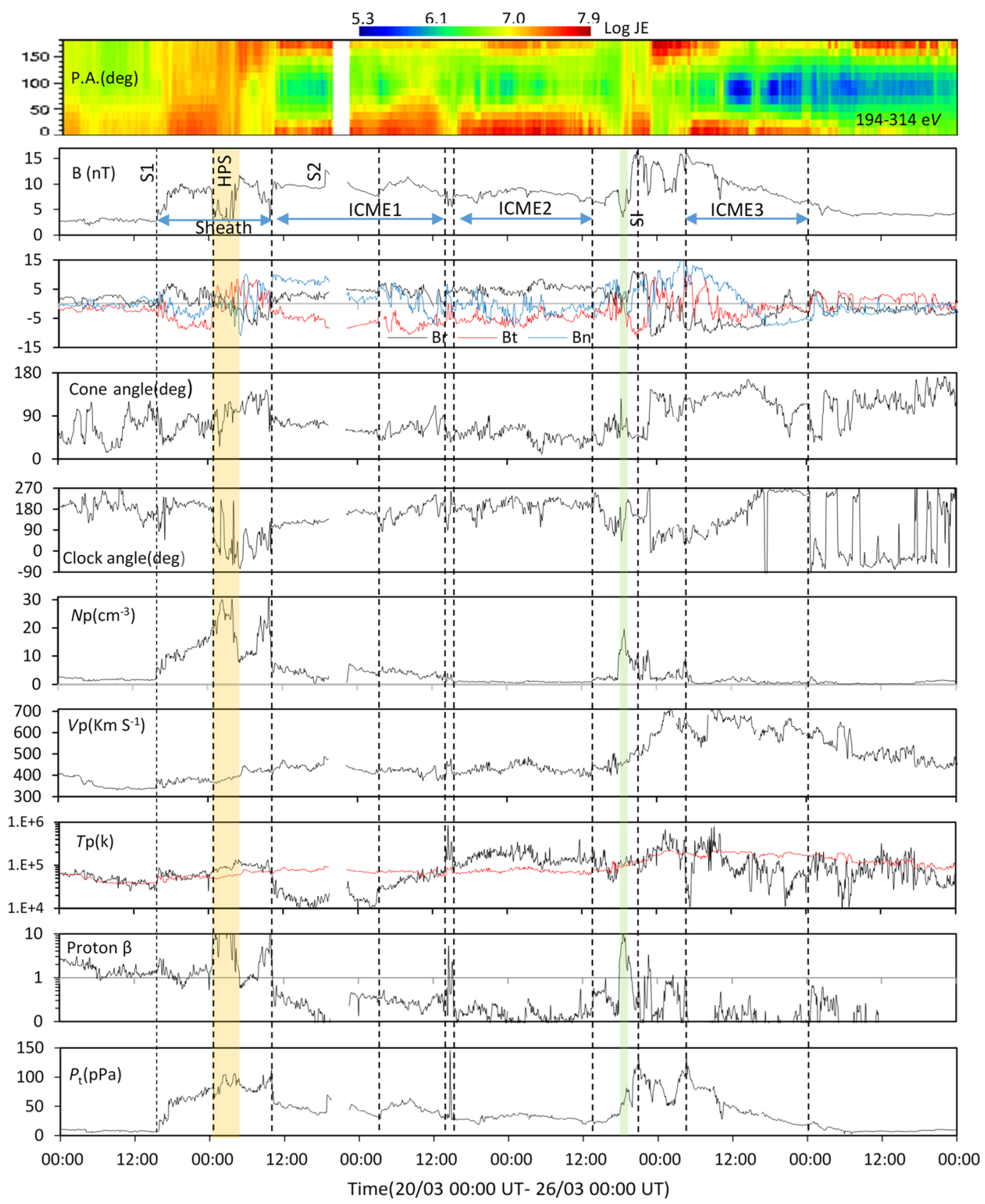

Fig. 5 The magnetic field and plasma parameters of the event. From top to bottom, the panels display the suprathermal electron pitch angle distribution (194-314 eV), the magnetic field strength B, Br, Bt, Bn, cone angle (deg), clock angle (deg), proton density $N_{p}\left(\mathrm{~cm}^{-3}\right)$, proton speed $V_{p}\left(\mathrm{~km} \mathrm{~s}^{-1}\right)$, proton temperature $T_{p}(\mathrm{~K})$ and expected temperature $T_{e x}(\mathrm{~K})$ (red line), solar wind $\beta_{\mathrm{p}}$, dynamic pressure $(\mathrm{nPa})$, and total perpendicular pressure $(\mathrm{pPa})$

temperature of ICME2 may support the penetration of S2 through ICME2. In addition, the location of the maximum Alfven speed which is close to the middle of ICME2, is likely to dissipate the coming shock from the fastest region (CIR/ICME3).

The shock weakens due to the large upstream Alfvenic speed $(250 \mathrm{~km} / \mathrm{s})$, an increase from about $150 \mathrm{~km} / \mathrm{s}$ from the trailing boundary to the center of ICME2. The proton speed compression and acceleration in many locations. The high 
Fig. 6 The Magnetic field and plasma parameters for the duration of March 20, 12:00 UT-March 24, 00:00 UT. From the upper panel: Magnetic field strength B, proton density $N_{p}$ $\left(\mathrm{cm}^{-3}\right)$, proton speed $V_{p}$ $\left(\mathrm{km} \mathrm{s}^{-1}\right)$, proton temperature $T_{p}$ $(\mathrm{K})$ and expected temperature $T_{e x}(\mathrm{~K})$ (red line), solar wind $\beta_{\mathrm{p}}$, entropy, Alfven speed $A_{v}$, Mach number $M_{A}$, dynamic pressure $P_{d y}(\mathrm{nPa})$, and $\mathrm{Pt}(\mathrm{pPa})$

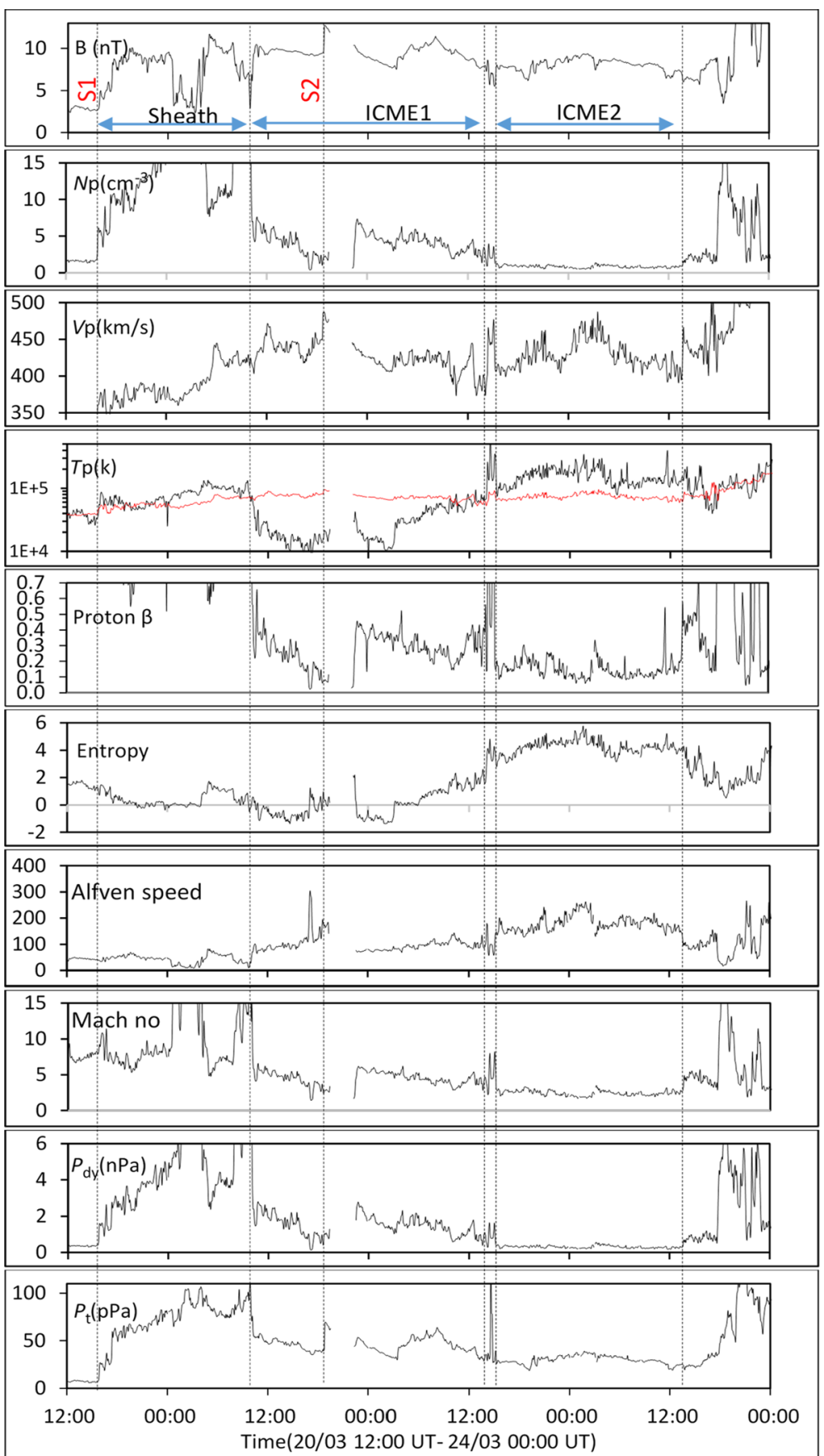

also increases by about $80 \mathrm{~km} / \mathrm{s}$, which is sufficient to ensure that the shock does not continue as a fast mode shock while it propagates. We interpret the speed profile of S2 as a magnetosonic pressure wave decaying from a magnetosonic shock due to the reduction of the local proton beta, and dissipating while advancing into ICME2.

However, the second scenario is inconsistent with the fact that S2 is $\sim 48$ hours ahead of ICME3, which may be too much of a delay for that shock to be driven by CME3. With the speed of the compound stream $(\sim 450 \mathrm{~km} / \mathrm{s})$, a time difference of 48 hours corresponds to approximately a $0.5 \mathrm{AU}$ spatial separation. A typical sheath width at $1 \mathrm{AU}$ is 0.08 AU (Richardson and Cane 2010). A third possibility is that S2 may be a shock remnant.

\section{ICMEs analysis}

The suprathermal electron pitch angle distribution displays a similar heat flux distribution along the three ICMEs (Fig. 5): a bidirectional behavior with a significant enhancement at 
$0^{\circ}$. The dominant suprathermal electron pitch angle direction for both MCs rotates with the magnetic field clock angle, consistent with the STB path close to the flank of both MCs (with both MC sides rooted at the Sun). The $\mathrm{P}_{\mathrm{t}}$ morphology supports the possibility of a spacecraft passage through the flanks (Russell et al. 2005). The non-smooth profile of the longitudinal magnetic field may be due to the legs of the ICME. As suggested by Owens (2016), the flux rope may be confined to the $\mathrm{MC}$ and not to its legs, even though the legs are magnetically connected with the Sun. Throughout the event, the only disappearance of the $0^{\circ}$ heat flux enhancement is during the short period before the ICME1 pressure wave, which implies that the pressure wave (which may of started as a forward shock driven by CIR/ICME3 and travelled through ICME2 and ICME1) plays a role in energizing the $0^{\circ}$ heat flux in every region that it penetrates, and that it rotates with the magnetic field. Due to this, S2 does not complete its passage through ICME1, and the upstream region's suprathermal electron distribution at both sides does not show any enhancement. The temperature of ICME2 is greater than ICME1. It may be due to the spacecraft trajectory (with respect to the MC axes) away from the MCs nose, or because ICME1 is an MC while ICME2 is an ejecta (non-MC ICME) (e.g. (Lepping et al. 2006; Riley and Richardson 2013)).

According to the 2 nd possible scenario, the pressure wave $\mathrm{S} 2$ has an effect on the $T_{p}$ profile. It is also possible that the passage of the forward shock driven by ICME3 increases compression. The shock reaches the pressure wave level with access to the rear ICME1 boundary, and this reduces the compression and is followed by plasma heating. Within ICME1 and after the pressure wave passes, $\beta_{\mathrm{p}}$ is between 0.15 and 0.5 , with an average value of 0.31 , whereas its average value is 0.15 throughout ICME2. The $\beta_{\mathrm{p}}$ profile supports the scenario of the shock path propagating through both ICMEs, causing a rapid and immediate effect on the $\beta_{\mathrm{p}}$ profile. This is apparent from the high value in the middle of ICME1 where S2 is located; with the move towards the rear of ICME1, $\beta_{p}$ declined gradually. The $\beta_{p}$ values during both ICME1 and ICME2 are higher than the typical isolated ICME $\beta_{\mathrm{p}}$ values.

Whilst this interpretation of the behaviour of S2 through ICME1 and ICME2 is valid, a strong counterargument is that $\mathrm{S} 2$ is $\sim 48$ hours ahead of ICME3.

\section{The embedded CIR and ICME3}

Figure 7 focuses on the merged CIR/ICME3 region. The first signature of this region is seen on March 23, 13:10 UT (see the 1 st vertical dotted line) accompanied by an increase in the solar wind speed, density, and $\beta_{\mathrm{p}}$, followed on March $23,17: 50$ UT by a sudden magnetic field magnitude drop (shaded green) associated with a sharp rise in $N_{p}$ (1.6 to $\left.19.6 \mathrm{~cm}^{-3}\right)$ and $\beta_{\mathrm{p}}(0.1$ to 8.2$)$, suggesting a magnetic reconnection exhaust region caused by a transference of magnetic energy into particle energy (Gosling et al. 2007). Following this region, the solar wind speed increases gradually, with a significant jump in the magnetic field strength and $\mathrm{P}_{\mathrm{t}}$ occurring on March 23, 17:45 UT (from 7.2 to $15.7 \mathrm{nT}$ ). There is also an enhancement in heat flux at $0^{\circ}$ associated with the CIR and indicating an SI at 20:55 UT. $\mathrm{P}_{\mathrm{t}}$ peaks and $N_{p}$ decreases, but there is no change in $T_{p}$ (see the 2 nd vertical dotted line).

The heat flux direction rotates with the magnetic field. A second magnetic field decrease occurs on March 23, 22:30 $\mathrm{UT}$, and is associated with decreasing density, increasing $\beta_{\mathrm{p}}$, and magnetic field cone and clock angle reversals. This suggests the existence of a HCS (see the 3rd vertical line) which matches a sector boundary crossing.

A third decrease in magnetic field can be seen on March 24, between 1:00 and 03:55 UT. Here the heat flux remains enhanced, with no significant rise in either density or $\beta_{\mathrm{p}}$, meaning that any magnetic reconnection exhaust is either weak or absent. This may be due to the symmetrical nature of the plasma at the boundaries of this region because of the embedded state. The ICME3 start time is close to 03:00 UT on May 24 (see the 4th vertical line), with a high elevation in the magnetic strength and $\mathrm{P}_{\mathrm{t}}$. The plasma profile properties before and during this time are complicated, especially during the preceding drop in the magnetic field. As mentioned before, this supports the probable symmetric nature of the boundary plasma as a result of the merger of the CIR and ICME3.

A second $P_{t}$ peak (see Fig. 7) is accompanied by a gradual proton speed reduction (see the 5th vertical dotted line). The solar wind parameters at this peak show a second potential stream interface SI2 location (maximum $P_{t}, T_{p}$ about to increase, shear velocity location). However, a sudden drop in $T_{p}$ to its lowest value (see the 6th vertical dotted line) occurs for a short three hour period, followed by a high increase for about five hours, associated with a large jump in $V_{p}$. The large temperature decrease at the 6th vertical dotted line is an indicator of the MC start time. This is associated with a decrease in $\beta_{\mathrm{p}}$, a strong counter-streaming heat flux, and a decline in proton speed. These effects are all due to the high expansion and deceleration of the $\mathrm{MC}$ in the fast wind side of the CIR as the structures interact. The MC clock angle (5th panel) starts with a small crank rotation for about 4.5 hours and becomes smooth until March 25, 00:45 UT, reaching an angle $>30^{\circ}$ for a duration of about 20 hours and confirming the existence of a MC flux rope within the CIR. The $B_{n}$ profile displays a smooth magnetic field with coherent rotation lasting from March 24, 06:05 (7th vertical dotted line) to March 24, 17:30 UT (8th vertical dotted line), lasting about 11.5 hours in total. The $B_{n}$ appears 


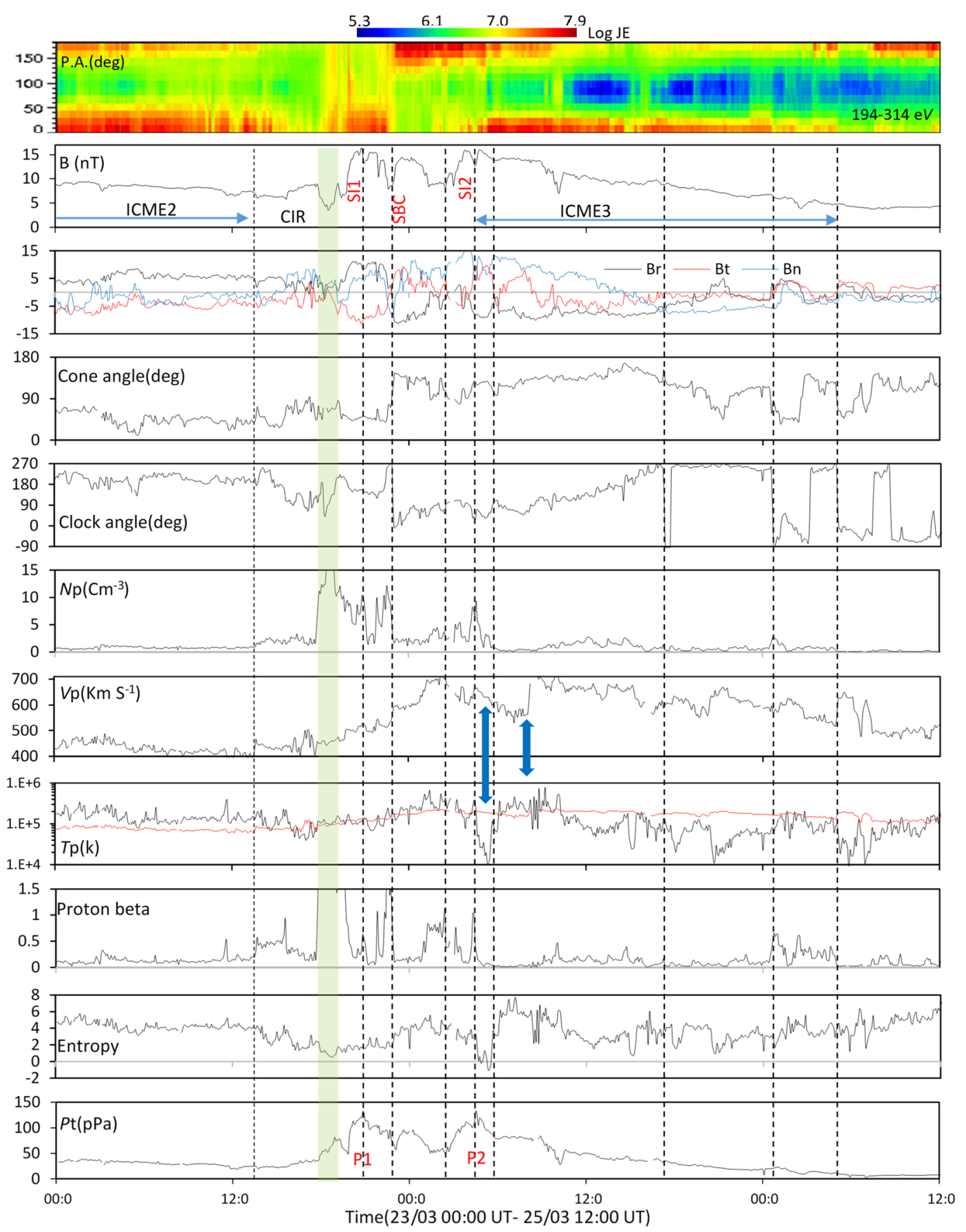

Fig. 7 The magnetic field and plasma parameters for the duration March 23, 00:00 UT-March 25, 12:00 UT. From top to bottom: the suprathermal electron pitch angle distribution (194-314 eV), magnetic field strength $\mathrm{B}, \mathrm{Br}, \mathrm{Bt}, \mathrm{Bn}$, cone angle (deg), Clock angle (deg), pro-

varied, but maintains its rotation until the 9th vertical dotted line on March 25, 00:50 UT. The clock angle rotation continues with a very short reversal at the 8 th vertical dotted line. However, this is a local reversal, because the dominant heat flux is sunward within the counter-streaming; this may be attributed to the large-scale helicity (Crooker et al. 1996). ton density $N_{p}\left(\mathrm{~cm}^{-3}\right)$, proton speed $V_{p}\left(\mathrm{~km} \mathrm{~s}^{-1}\right)$, proton temperature $T_{p}(\mathrm{~K})$ and expected temperature $T_{e x}(\mathrm{~K})$ (red line), solar wind $\beta_{\mathrm{p}}$, entropy, dynamic pressure $(\mathrm{nPa})$, and total perpendicular pressure $(\mathrm{pPa})$

\section{The impact of the ICME3/CIR merger}

ICME3 has an impact on both the CIR speed and $T_{p}$ profile. The sudden appearance of ICME3 within the fast wind side of the CIR causes the temperature (which would usually be expected to increase after SI2 in the absence of interaction with the MC) to drop suddenly to its lowest level in about 1.2 
hours, from $3.89 \times 10^{5} \mathrm{~K}$ to $1.07 \times 10^{4} \mathrm{~K}$ (by a factor of 36 ). Furthermore, the fast solar wind speed is reduced during its expansion and deceleration within this area (see the 1st blue heavy straight line in Fig. 7). The impact of the CIR/ICME3 interaction include the high $T_{p}$ elevation following the initial large reduction (Fig. 7, the 2nd blue heavy straight line) and the large increase in solar wind speed. The ICME3 temperature is higher than expected due to the high-speed acceleration and its position within this hot, high-speed stream. Another relevant factor is the location of a second SI (SI2) within the MC. The presence of an SI is supported by a clear increase in entropy, accompanied by a decrease in proton density and an increase in $T_{p}$, which are typical SI criteria. After about five hours the MC temperature decreases as a result of its deceleration and expansion towards the final boundary, but remains higher than typical MC temperatures in most regions $\left(T_{p}<0.5 T_{e x}\right)$. The fast wind which follows the CIR influences not only the ICME3 temperature but also its $\beta_{p}$. The average $\beta_{p}$ value of ICME3 is 0.16 with a peak of 0.4 , which is a little higher than typical values for the MC $(0.06 \pm 0.04)$ (Farrugia et al. 1993; Burlaga et al. 2001). The entropy is also unstable compared to that of the two previous ICMEs. The weakness of the magnetic reconnection exhaust region within the CIR/ICME shows that the two structures are embedded. It also suggests that the interaction between the two wide, coherent $\mathrm{P}_{t}$ peaks ( $\mathrm{P} 1$ and $\mathrm{P} 2$ ) is greater and more complicated than the ICME1-ICME2 interaction within the same compound stream.

\section{Discussion and conclusions}

The in-situ measurements taken by STEREO B reveal three ICMEs or interplanetary ejecta. These findings are difficult to reconcile with remote sensing observations. The results suggest either that ICME2 is a compressed interplanetary ejecta region between ICME1 and CIR/ICME3, or that there were originally three CMEs, but due to the complexity and the position of the active region, the remote sensing images were unable to display the sequences of events clearly. The in-situ data at $1 \mathrm{AU}$ shows a penetration of a forward pressure wave driven by ICME3/CIR, associated with a rise in $T_{p}$ throughout the second ICME and in part of the first one. The third ICME is merged with and embedded in a CIR. The lack of magnetic energy conversion into particle energy at the magnetic reconnection exhaust region between the CIR and ICME3 supports the interpretation that these are an embedded structure.

Throughout the event, the proton speed profile does not exceed $450 \mathrm{~km} / \mathrm{s}$ within ICME1 and ICME2, except for a few regions, whereas it matches the compound stream speed criteria within ICME3. This means that the compound stream may experience low speed. The understanding of general criteria of observational characteristics, including the stream speed in compound streams, will depend on the future study of similar events.

The magnetic reconnection exhaust region between ICME1 and ICME2 shows that they are separate structures with distinct region boundaries, and that they are two different interplanetary ejecta. This exhaust region is weaker than its counterpart between the ICME2 rear boundary and ICME3/CIR, possibly due to the asymmetric distribution of plasma between the ICME2 rear boundary and the CIR front boundary.

The appearance of the first HPS within the sheath is a temporary change of the interplanetary magnetic field sector boundary during the passage of ICME1. The ICME therefore disrupts the coronal streamer belt locally. Whilst a reverse shock is expected to follow ICME2 as its speed is decreased due to ICME1, none is present in this case. This may be due to the dissipation and decay of the second shock (S2). A significant appearance of a SI within the ICME3 magnetic flux rope suggests that the SI has penetrated the flux rope and has changed its characteristics. The sudden appearance of ICME3 within the fast wind side of the CIR causes $T_{p}$ to drop suddenly to its lowest level in about 1.2 hours. ICME3 impacts the CIR through expansion and deceleration.

The suprathermal electron pitch angle distribution throughout the event displays an absence of interchange reconnection, although there is an interaction between open and closed magnetic field structures (CIR and ICMEs). This result implies that the CIR magnetic field direction is inwards during the interaction with the ICME inward flank which is not conducive to reconnection.

Acknowledgements We are grateful to Lan K. Jian, Skoug, Ruth M, Benoit Lavraud, Emmanuel Penou and Noe' Lugaz for helpful comments. The solar wind magnetic field and plasma data are courtesy of the STEREO MAG, IMPACT and SWE teams. Morgan acknowledges the support of STFC grant ST/S000518/1 (UK) to Aberystwyth University.

Publisher's Note Springer Nature remains neutral with regard to jurisdictional claims in published maps and institutional affiliations.

Open Access This article is licensed under a Creative Commons Attribution 4.0 International License, which permits use, sharing, adaptation, distribution and reproduction in any medium or format, as long as you give appropriate credit to the original author(s) and the source, provide a link to the Creative Commons licence, and indicate if changes were made. The images or other third party material in this article are included in the article's Creative Commons licence, unless indicated otherwise in a credit line to the material. If material is not included in the article's Creative Commons licence and your intended use is not permitted by statutory regulation or exceeds the permitted use, you will need to obtain permission directly from the copyright holder. To view a copy of this licence, visit http://creativecommons.org/licenses/by/4.0/. 


\section{References}

Acuña, M., Curtis, D., Scheifele, J., Russell, C., Schroeder, P., Szabo, A., Luhmann, J.: The STEREO/IMPACT magnetic field experiment. Space Sci. Rev. 136, 203-226 (2008)

Al-Shakarchi, D.A.M.: A multi-spacecraft study of interacting ICMEs and CIRs in interplanetary space. Aberystwyth University (2018)

Al-Shakarchi, D.A., Morgan, H.: Properties of the HPS-ICME-CIR interaction event of 9-10 September 2011. J. Geophys. Res. Space Phys. 123, 2535-2556 (2018)

Brueckner, G., Howard, R., Koomen, M., Korendyke, C., Michels, D., Moses, J., Socker, D., Dere, K., Lamy, P., Llebaria, A.: The large angle spectroscopic coronagraph (LASCO). Sol. Phys. 162, 357402 (1995)

Burlaga, L.: Interplanetary streams and their interaction with the Earth. Space Sci. Rev. 17, 327-352 (1975)

Burlaga, L., Ogilvie, K.: Solar wind temperature and speed. Astrophys. Space Sci. 16, 465 (1972)

Burlaga, L., Mcdonald, F., Schwenn, R.: Formation of a compound stream between $0.85 \mathrm{AU}$ and 6.2 AU and its effects on solar energetic particles and galactic cosmic rays. J. Geophys. Res. Space Phys. 91, 13331-13340 (1986)

Burlaga, L., Behannon, K., Klein, L.: Compound streams, magnetic clouds, and major geomagnetic storms. J. Geophys. Res. Space Phys. 92, 5725-5734 (1987)

Burlaga, L., Skoug, R., Smith, C., Webb, D., Zurbuchen, T., Reinard, A.: Fast ejecta during the ascending phase of solar cycle 23: ACE observations, 1998-1999. J. Geophys. Res. Space Phys. 106, 20957-20977 (2001)

Burlaga, L., Plunkett, S., St Cyr, O.: Successive CMEs and complex ejecta. J. Geophys. Res. Space Phys. 107, A10 (2002)

Cane, H., Richardson, I.: Interplanetary coronal mass ejections in the near-Earth solar wind during 1996-2002. J. Geophys. Res. Space Phys. 108, 1156 (2003)

Crooker, N., Burton, M., Siscoe, G., Kahler, S., Gosling, J., Smith, E.: Solar wind streamer belt structure. J. Geophys. Res. Space Phys. 101, 24331-24341 (1996)

Farrugia, C., Berdichevsky, D.: Evolutionary signatures in complex ejecta and their driven shocks. Ann. Geophys. 22, 3679-3698 (2004)

Farrugia, C., Burlaga, L., Osherovich, V., Richardson, I., Freeman, M., Lepping, R., Lazarus, A.: A study of an expanding interplanetary magnetic cloud and its interaction with the Earth's magnetosphere: the interplanetary aspect. J. Geophys. Res. Space Phys. 98, 7621-7632 (1993)

Farrugia, C., Jordanova, V., Thomsen, M., Lu, G., Cowley, S., Ogilvie, K.: A two-ejecta event associated with a two-step geomagnetic storm. J. Geophys. Res. Space Phys. 111, A11 (2006)

Farrugia, C., Kilpua, E., Jian, L., Liu, Y., Eastwood, J., Harrison, R., Webb, D., Temmer, M., Odstrcil, D., Davies, J.: Multi-point shock and flux rope analysis of multiple interplanetary coronal mass ejections around 2010 August 1 in the inner heliosphere. Astrophys. J. 758, 10 (2012)

Forsyth, R., Bothmer, V., Cid, C., Crooker, N., Horbury, T., Kecskemety, K., Klecker, B., Linker, J., Odstrcil, D., Reiner, M.: ICMEs in the Inner Heliosphere: Origin, Evolution and Propagation Effects. Coronal Mass Ejections. Springer, Berlin (2006)

Gosling, J., Eriksson, S., Mccomas, D., Phan, T., Skoug, R.: Multiple magnetic reconnection sites associated with a coronal mass ejection in the solar wind. J. Geophys. Res. Space Phys. 112, A08106 (2007)

Harrison, R., Davies, J., Möstl, C., Liu, Y., Temmer, M., Bisi, M., Eastwood, J., De Koning, C., Nitta, N., Rollett, T.: An analysis of the origin and propagation of the multiple coronal mass ejections of 2010 August 1. Astrophys. J. 750, 45 (2012)
Howard, R., Moses, J., Socker, D., Dere, K., Cook, J., Consortium, S.: Sun Earth connection coronal and heliospheric investigation (SECCHI). Adv. Space Res. 29, 2017-2026 (2002)

Jian, L., Russell, C., Luhmann, J., Skoug, R., Steinberg, J.: Stream interactions and interplanetary coronal mass ejections at 5.3 AU near the solar ecliptic plane. Sol. Phys. 250, 375-402 (2008)

Jian, L., Russell, C., Luhmann, J., Galvin, A., Simunac, K., Zank, G.P., Borovsky, J., Bruno, R., Cirtain, J., Cranmer, S.: Solar wind observations at STEREO: 2007-2011. In: AIP Conference Proceedings, pp. 191-194. AIP, New York (2013)

Kaiser, M.: The STEREO mission: an overview. Adv. Space Res. 36, 1483-1488 (2005)

Lepping, R., Berdichevsky, D., Wu, C.-C., Szabo, A., Narock, T., Mariani, F., Lazarus, A., Quivers, A.: A summary of WIND magnetic clouds for years 1995-2003: model-fitted parameters, associated errors and classifications. Ann. Geophys. 24, 215-245 (2006)

Liu, Y.D., Luhmann, J.G., Möstl, C., Martinez-Oliveros, J.C., Bale, S.D., Lin, R.P., Harrison, R.A., Temmer, M., Webb, D.F., Odstrcil, D.: Interactions between coronal mass ejections viewed in coordinated imaging and in situ observations. Astrophys. J. Lett. 746, L15 (2012)

Liu, Y.D., Hu, H., Wang, R., Yang, Z., Zhu, B., Liu, Y.A., Luhmann, J.G., Richardson, J.D.: Plasma and magnetic field characteristics of solar coronal mass ejections in relation to geomagnetic storm intensity and variability. Astrophys. J. Lett. 809, L34 (2015)

Lopez, R.: Solar cycle invariance in solar wind proton temperature relationships. J. Geophys. Res. 92, 11189-11194 (1987)

Lugaz, N., Manchester Iv, W., Gombosi, T.: Numerical simulation of the interaction of two coronal mass ejections from Sun to Earth. Astrophys. J. 634, 651 (2005)

Lugaz, N., Farrugia, C., Davies, J., Möstl, C., Davis, C., Roussev, I., Temmer, M.: The deflection of the two interacting coronal mass ejections of 2010 May 23-24 as revealed by combined in situ measurements and heliospheric imaging. Astrophys. J. 759, 68 (2012)

Lugaz, N., Farrugia, C., Smith, C., Paulson, K.: Shocks inside CMEs: a survey of properties from 1997 to 2006. J. Geophys. Res. Space Phys. 120, 2409-2427 (2015)

Lugaz, N., Temmer, M., Wang, Y., Farrugia, C.: The interaction of successive coronal mass ejections: a review. Solar Phys. 292, 64 (2017)

Luhmann, J., Curtis, D., Schroeder, P., Mccauley, J., Lin, R., Larson, D., Bale, S., Sauvaud, J.-A., Aoustin, C., Mewaldt, R.: STEREO IMPACT investigation goals, measurements, and data products overview. Space Sci. Rev. 136, 117-184 (2008)

Maričić, D., Vršnak, B., Dumbović, M., Žic, T., Roša, D., Hržina, D., Lulić, S., Romštajn, I., Bušić, I., Salamon, K.: Kinematics of interacting ICMEs and related Forbush decrease: case study. Sol. Phys. 289, 351-368 (2014)

Mishra, W., Srivastava, N., Chakrabarty, D.: Evolution and consequences of interacting CMEs of 9-10 November 2012 using STEREO/SECCHI and in situ observations. Sol. Phys. 290, 527$552(2015)$

Morgan, H.: An atlas of coronal electron density at $5 \mathrm{R} \odot$. I. Data processing and calibration. Astrophys. J. Suppl. Ser. 219, 23 (2015)

Morgan, H., Druckmüller, M.: Multi-scale Gaussian normalization for solar image processing. Sol. Phys. 289, 2945-2955 (2014)

Morgan, H., Habbal, S.R.: Observational aspects of the threedimensional coronal structure over a solar activity cycle. Astrophys. J. 710, 1 (2010)

Morgan, H., Habbal, S.R., Woo, R.: The depiction of coronal structure in white-light images. Sol. Phys. 236, 263-272 (2006)

Morgan, H., Byrne, J.P., Habbal, S.R.: Automatically detecting and tracking coronal mass ejections. I. Separation of dynamic and quiescent components in coronagraph images. Astrophys. J. 752, 144 (2012) 
Morgan, H., Jeska, L., Leonard, D.: The expansion of active regions into the extended solar corona. Astrophys. J. Suppl. Ser. 206, 19 (2013)

Oliveros, J.C.M., Raftery, C.L., Bain, H.M., Liu, Y., Krupar, V., Bale, S., Krucker, S.: The 2010 August 1 type II burst: a CME-CME interaction and its radio and white-light manifestations. Astrophys. J. 748, 66 (2012)

Owens, M.: Do the legs of magnetic clouds contain twisted flux-rope magnetic fields? Astrophys. J. 818, 197 (2016)

Prise, A., Harra, L., Matthews, S., Arridge, C.S., Achilleos, N.: Analysis of a coronal mass ejection and corotating interaction region as they travel from the Sun passing Venus, Earth, Mars, and Saturn. J. Geophys. Res. Space Phys. 120, 1566-1588 (2015)

Richardson, I., Cane, H.: Regions of abnormally low proton temperature in the solar wind (1965-1991) and their association with ejecta. J. Geophys. Res. Space Phys. 100, 23397-23412 (1995)

Richardson, I., Cane, H.: Near-Earth interplanetary coronal mass ejections during solar cycle 23 (1996-2009): catalog and summary of properties. Sol. Phys. 264, 189-237 (2010)

Riley, P., Richardson, I.: Using statistical multivariable models to understand the relationship between interplanetary coronal mass ejecta and magnetic flux ropes. Sol. Phys. 284, 217-233 (2013)

Russell, C., Shinde, A., Jian, L.: A new parameter to define interplanetary coronal mass ejections. Adv. Space Res. 35, 2178-2184 (2005)

Sauvaud, J.-A., Larson, D., Aoustin, C., Curtis, D., Medale, J.-L., Fedorov, A., Rouzaud, J., Luhmann, J., Moreau, T., Schröder, P.: The IMPACT Solar Wind Electron Analyzer (SWEA). The STEREO Mission. Springer, Berlin (2008)
Shen, C., Wang, Y., Wang, S., Liu, Y., Liu, R., Vourlidas, A., Miao, B., Ye, P., Liu, J., Zhou, Z.: Super-elastic collision of large-scale magnetized plasmoids in the heliosphere. Nat. Phys. 8, 923-928 (2012)

Temmer, M., Vršnak, B., Rollett, T., Bein, B., De Koning, C.A., Liu, Y., Bosman, E., Davies, J.A., Möstl, C., Žic, T.: Characteristics of kinematics of a coronal mass ejection during the 2010 August 1 CME-CME interaction event. Astrophys. J. 749, 57 (2012)

Temmer, M., Veronig, A., Peinhart, V., Vršnak, B.: Asymmetry in the CME-CME interaction process for the events from 2011 February 14-15. Astrophys. J. 785, 85 (2014)

Wang, Y.M., Ye, P.Z., Wang, S.: Multiple magnetic clouds: several examples during March-April. J. Geophys. Res. Space Phys. 108, 1370 (2003). 2001

Winslow, R.M., Lugaz, N., Schwadron, N.A., Farrugia, C.J., Yu, W., Raines, J.M., Mays, M.L., Galvin, A.B., Zurbuchen, T.H.: Longitudinal conjunction between MESSENGER and STEREO a: development of ICME complexity through stream interactions. J. Geophys. Res. Space Phys. 121, 6092-6106 (2016)

Xu, X., Wang, Y., Wei, F., Feng, X., Deng, X., Ma, Y., Zhou, M., Pang, Y., Wong, H.-C.: Direct evidence for kinetic effects associated with solar wind reconnection. Sci. Rep. 5, 8080 (2015)

Zhao, X., Hoeksema, J.T.: Effect of coronal mass ejections on the structure of the heliospheric current sheet. J. Geophys. Res. Space Phys. 101, 4825-4834 (1996)

Zurbuchen, T.H., Richardson, I.G.: In-situ solar wind and magnetic field signatures of interplanetary coronal mass ejections. Space Sci. Rev. 123, 31-43 (2006) 